Pacific Journal of Mathematics

TOTALLY REAL REPRESENTATIONS AND REAL FUNCTION
SPACES 


\section{TOTALLY REAL REPRESENTATIONS AND REAL FUNCTION SPACES}

\section{Calvin C. Moore AND Joseph A. Wolf}

Let $G$ be a locally compact group. The notion of "totally real" unitary representation of $G$ is defined and investigated in $\S 1$. In particular, if $K$ is a compact subgroup of $G$, it is shown that every closed $G$-invariant subspace of $L_{2}(G / K)$ is spanned by real-valued functions if, and only if, $K g K=K g^{-1} K$ for every $g \in G$. In $\$ 2$ the coset space $X=G / K$ is specialized to a Riemannian symmetric space, where the double coset condition is replaced by a simple Weyl group condition.

o. Introduction. Let $X$ be a riemannian symmetric space of compact type and $G$ its largest connected group of isometries. In his 1929 paper [1] on class 1 representations, É. Cartan showed that the symmetry of $X$ sends every uniformly closed $G$-invariant function space on $X$ to its complex conjugate. Starting from the point of view of algebras, Mirkil and de Leeuw [4] showed that every rotation invariant function algebra on the sphere $S^{n}(n \geqq 2)$ was spanned by real-valued functions, hence (Stone-Weierstrass theorem) that such an algebra necessarily was all continuous functions on $S^{n}$, all continuous functions on real projective $n$-space, or just the constantsthat state of affairs is quite different from the case $n=1$. When the rotation group $S O(n+1)$ contains the symmetry of $S^{n}$, i.e. when $n$ is even, Cartan's result mentioned above implies reality of such function algebras. The published Mirkil-de Leeuw argument rests rather on the fact that the spherical harmonics are real-valued.

The Cartan and Mirkil-de Leeuw results were unified when I. Glicksberg and one of us found a general result [12, Theorem 2.1] on G-invariant function spaces on compact symmetric spaces, formulated in terms of the double coset condition mentioned in the Abstract. One of us then translated the double coset condition into an easily-checked Weyl group condition [12, Theorem 5.1] and extended the Mirkil-de Leeuw result on function algebras [12, Theorem 7.1]. That translation made essential use of É. Cartan's classification of symmetric spaces, and was later freed of the classification by J. A. Tirao [9].

We discuss this circle of problems for coset spaces $X=G / K, G$ locally compact and $K$ compact. Although the idea is very much the same, the proofs are more streamlined and are freed of many differentiability and compactness restrictions. Until now, however, our only significant applications are to Riemannian symmetric spaces. 
1. Let $\pi$ be a unitary representation of a locally compact group $G$ on a (complex) Hilbert space $H$. Let $H_{R}$ be a real form of $H$; that is $H=H_{R} \oplus i H_{R}$ as real Hilbert spaces. We say that $H_{R}$ is invariant if $\pi(g) H_{R}=H_{R} \forall g \in G$, and that $\pi$ is totally real relative to $H_{R}$ if in addition $H_{R} \cap V$ is a real form of $V$ for every closed $G$-invariant subspace $V \subset H$. If one thinks of $H_{R}$ as "real" elements, the condition says that $G$ preserves real elements, and moreover, that every invariant subspace is spanned by the real elements in it. Now suppose that $K$ is a compact subgroup of $G$, and that $\pi$ is the natural representation by left translation on $H=L_{2}(G / K)$. The real valued functions in $H$ constitute an invariant real form, and the circle of problems discussed in the introduction is more or less the same as determining when $\pi$ is totally real. This we will do below. Although the ideas involved have been known for some years in many contexts (see for instance [2], [5], [6], [8], [10], [11]), it nevertheless seems worthwhile to present them again in the precise form needed.

$A_{K}$ will denote the convolution algebra of all continuous functions with compact support on $G$ which are biinvariant under $K$, i.e., $f\left(k g k_{2}\right)=f(g)$. Then $A_{K}$ is a subalgebra of $L_{1}(G)$ and inherits the involution $f \rightarrow f^{*}, f^{*}(x)=\overline{f\left(x^{-1}\right)} \Delta\left(x^{-1}\right)$, where $\Delta$ is the modular function of $G$.

THEOREM 1. Let $G$ be a locally compact group, $K$ a compact subgroup, then the following are equivalent.

(1) Every closed G-invariant subspace of $L_{2}(G / K)$ is spanned by real functions

(2) The representation $\pi$ of $G$ on $L_{2}(G / K)$ is totally real relative to some invariant real form

(3) $\pi$ is totally real relative to any invariant real form

(4) $f^{*}=\bar{f}$ for $f \in A_{K}$

(5) $K g^{-1} K=K g K, \forall g \in G$

(6) $K g^{-1} K=K g K$ for almost all $g \in G$.

Under the above conditions, $G$ is unimodular and $\pi$ is multiplicity free.

Let $\pi$ be a unitary representative of $G$ on a Hilbert space $H$. If $H_{R}$ is any real form, there exists a unique conjugate linear isometric involution $\nu$ having $H_{R}$ as its fixed points, and conversely every such $\nu$ determines a real form. Evidently $H_{R}$ is invariant if and only if $\nu$ commutes with all the operators $\pi(g)$, in which case we say that $(\pi, \nu)$ is real, and moreover, $\pi$ is totally real relative to $H_{R}$ if and only if in addition $\nu$ commutes with the orthogonal projection onto every $G$-invariant subspace, in which case we say that $(\pi, \nu)$ is totally real.

$C(\pi)$ denotes the commuting algebra of $\pi$; it is the von Neumann 
algebra of all bounded operators on $H$ commuting with all the $\pi(g)$. Let $(\pi, \nu)$ be real; then $A \in C(\pi)$ implies $A^{\nu}=\nu A \nu^{-1}=\nu A \nu \in C(\pi)$ and $A \rightarrow A^{\nu}$ is a conjugate linear automorphism of $C(\pi)$.

Lemma 1. Let $(\pi, \nu)$ be real. Then $(\pi, \nu)$ is totally real if and only if $A^{\nu}=A^{*}$ for all $A \in C(\pi)$.

Proof. If $A^{\nu}=A^{*}$, and if $P$ is the projection onto any $\pi$ invariant subspace, $P=P^{*}$, and so $P=P^{\nu}$ so that $\pi$ is totally real. If $\pi$ is totally real, then $P^{\nu}=P=P^{*}$ for any such $P$, and since every $A$ in $C(\pi)$ is a norm limit of linear combinations of such projections, we evidently have $A^{*}=A^{\nu}$, completing the proof.

Now if $(\pi, \nu)$ is totally real, $C(\pi)$ is necessarily commutative since $B^{*} A^{*}=(A B)^{*}=(A B)^{\nu}=A^{\nu} B^{\nu}=A^{*} B^{*}$. Thus $\pi$ is multiplicity free (locally simple) by definition. Moreover if $\mu$ is another involution such that $(\pi, \mu)$ is real, it is immediate that $\mu=\nu U$ for some unitary operator $U$ in $C(\pi)$. Since $C(\pi)$ is commutative, a computation shows that $A^{\mu}=A^{\nu}=A^{*}$ so that $(\pi, \mu)$ is also totally real. This shows that conditions (1), (2) and (3) of the theorem are equivalent, and are in turn equivalent to $A^{*}=A^{\nu}$ in $C(\pi)$ for one (all) $\nu$. These arguments show that the notion total reality is independent of choice of invariant real form.

Proof of Theorem 1. We now fix $\nu$ to be complex conjugation of functions on $H=L_{2}(G / K)$, and we embbed $H$ as the subspace of $L_{2}(G)$ consisting of right $K$-invariant functions. Then if $f \in A_{K}$, the formula $\rho(f) h=h * f, h \in H$, defines a bounded operator in $C(\pi)$. Moreover $\rho$ is an injective anti-homomorphism of $A_{K}$ into $C(\pi)$; and $\rho(f)^{*}=\rho\left(f^{\prime}\right)$ where $f^{\prime}(x)=\overline{f\left(x^{-1}\right)}$. (Note the absence of the modular function.) A simple calculation shows that $\rho(f)^{\nu}=\rho(\bar{f})$, and so if $(\pi, \nu)$ is totally real we see that $\rho\left(f^{\prime}\right)=\rho(\bar{f})$. Since $\rho$ is injective, it follows that $f^{\prime}=\bar{f}$ or in other words $f(g)=f\left(g^{-1}\right)$. Since $A_{K}$ separates the double cosets of $K$ we have $K g^{-1} K=K g K$ for all $g$, so we have established (5).

An elementary continuity argument which we omit shows that (5) and (6) are equivalent. Moreover if (5) holds, then we have for every $g, g^{-1}=k_{1} g k_{2}$, and hence $\Delta(g)^{2}=1$ since the modular function is 1 on compact subgroups. Since $\Delta(g)>0, \Delta(g)=1$ so $G$ is unimodular, and now (4) follows from (5). Conversely suppose that (4) holds; if $g \in G$ we construct $f \in A_{K}$ such that $f(g)=f\left(g^{-1}\right) \neq 0$. Since $f^{*}=\bar{f}$, it follows that $\Delta(g)=1$ so that $G$ is unimodular, and now (5) follows from (4) so that (4), (5), and (6) are equivalent.

Finally suppose that (4) holds; since $G$ is unimodular we have 
$f^{\prime}=f^{*}$ and $\rho(f)^{\nu}=\rho(\bar{f})=\rho\left(f^{*}\right)=\rho\left(f^{\prime}\right)=\rho(f)^{*}$. As $G$ is unimodular, it follows from the results in Segal [7] or Kleppner [3] that the range of $\rho$ is weakly dense in $C(\pi)$. Thus we have $A^{\nu}=A^{*}$ for a weakly dense subset of $C(\pi)$, and hence $A^{\nu}=A^{*}$ for all $A$. Thus $(\pi, \nu)$ is totally real, completing the argument.

2. Application to symmetric spaces. We will apply Theorem 1 to a connected symmetric coset space $X=G / K$, say with symmetry $\sigma$. Thus $G$ is a Lie group, $K$ is a compact subgroup that meets every component of $G$, and $\sigma$ is an involutive automorphism of $G$ such that $K$ is a subgroup of finite index in the fixed point set $G^{\sigma}$. Let $\mathfrak{f} \subset \mathfrak{g}$ denote the Lie algebras of $K \subset G$ and $\mathfrak{g}=\mathfrak{H}+\mathfrak{m}$ the "Cartan decomposition" into $( \pm 1)$ - eigenspaces of $\sigma$. Recall that Cartan subalgebra of $(\mathfrak{g}, \mathfrak{f})$ means maximal object among the subalgebras of $\mathfrak{g}$ contained in $\mathfrak{m}$, and that Cartan subalgebras are commutative because $[m, m] \cap m=0$. Choose a Cartan subalgebra $a$ of $(\mathfrak{g}, \mathfrak{f})$. Recall that the Weyl group of $(G, K)$ relative to $a$ is the group

$$
W_{G, K}=\{k \in K: \operatorname{ad}(k) a=a\} /\{k \in K: \operatorname{ad}(k) \alpha=\alpha \text { for every } \alpha \in \mathfrak{a}\}
$$

of linear transformations of $a$; it is finite if and only if $G$ is reductive. If $\mathfrak{a}^{\prime}$ is another Cartan subalgebra of $(\mathfrak{g}, \mathfrak{f})$ and $W_{G, K}^{\prime}$ the corresponding Weyl group, then some $k \in K$ satisfies $a d(k) \mathfrak{a}^{\prime}=a$, so $\operatorname{ad}(k) W_{G, K}^{\prime}=W_{G, K}$.

Theorem 2. Let $X=G / K$ connected symmetric coset space and $W_{G, K}$ the Weyl group relative to a Cartan subalgebra a of $(\mathfrak{g}, \mathfrak{f})$. Then every closed $G$-invariant subspace of $L_{2}(X)$ is spanned by realvalued functions if, and only if, given $\alpha \in a$ there exists $w \in W_{G, K}$ such that $w(\alpha)=-\alpha$.

Proof. Suppose that every element of $a$ is sent to its negative by an element of $W_{G, K}$. Given $g \in G$ decompose $g=k_{1} \cdot \exp (\alpha) \cdot k_{2}$ where $\alpha \in \mathfrak{a}$ and $k_{i} \in K$. Choose $w \in W_{G, K}$ with $w(\alpha)=-\alpha$ and represent $w=a d(k), k \in K$. Then $K g K=K \exp (\alpha) K=K k \exp (\alpha) k^{-1} K=$ $K \exp (w \alpha) K=K \exp (-\alpha) K=K g^{-1} K$.

Conversely suppose $K g K=K g^{-1} K$ for every $g \in G$. If $a \in \exp (\mathfrak{a})$ now $a=k_{1} a^{-1} k_{2}, k_{i} \in K$; write that as $a=a d\left(k_{1}\right) a^{-1} \cdot k_{1} k_{2}$; for $a$ near 1 in $G$ it follows that $a=a d\left(k_{1}\right) a^{-1}$ and $k_{1} k_{2}=1$. If $\alpha \in \mathfrak{a}$ is near 0 now $a d(k) \alpha=-\alpha$ for some $k \in K$; the condition "near 0 " may be dropped by linearity, and $a d(k)$ may be replaced by some $w \in W_{G, K}$ because $a d(K)$-conjugacy on a forces $W_{G, K}$-conjugacy. Now every $\alpha \in \mathfrak{a}$ is sent to its negative by some $w \in W_{G, K}$. 
Theorem 2 now follows directly from Theorem 1 .

If $w \in W_{G, K}$ we have its $(-1)$-eigenspace $a_{w}$ on $a$. The criterion of Theorem 2 is that $a$ be the union of the $a_{w}$. If $W_{G, K}$ is finite then the union of the $\mathfrak{a}_{w}$ is a lower-dimensional set in $\mathfrak{a}$, unless $\mathfrak{a}_{w}=\mathfrak{a}$ for some $w$. Thus:

Corollary 2.1. Let $X=G / K$ connected symmetric coset space and $W_{G, K}$ the Weyl group relative to a Cartan subalgebra a of $(\mathfrak{g}, \mathfrak{k})$. Suppose that the Lie group $G$ is reductive. Then every closed Ginvariant subspace of $L_{2}(X)$ is spanned by real-valued functions if, and only if, $W_{G, K}$ contains the transformation $-I$ of $a$.

Let $X$ be a Riemannian symmetric space. Given $x \in X$ we represent $X$ as a connected symmetric coset space $G / K$ where $G$ is the largest connected group of isometries and $K$ is the stability subgroup at $x$. Then we write $W_{X}$ for $W_{G, K}$ and call it the Weyl group of $X$. Let $p: \bar{X} \rightarrow X$ be the universal Riemannian covering. Then $\bar{X}=X_{0} \times$ $X_{1} \times \cdots \times X_{r}$ (de Rham decomposition) where $X_{0}$ is an Euclidean space and the other $X_{i}$ are simply connected irreducible Riemannian symmetric spaces. We call the $X_{i}, 1 \leqq i \leqq r$, the irreducible local factors of $X$. Theorem 2 and the covering space methods of [13, §8.3] give us:

CoRollary 2.2. Let $X$ be a Riemannian symmetric space. $G$ the largest connected group of isometries of $X$, and $\left\{W_{1}, \cdots, W_{r}\right\}$ the Weyl groups of the irreducible local factors of $X$. Then every closed $G$-invariant subspace of $L_{2}(X)$ is spanned by real-valued functions if, and only if,

(i) the fundamental group $\pi_{1}(X)$ is finite, and

(ii) each $W_{i}, 1 \leqq i \leqq r$, contains the transformation $-I$.

Condition (i) of Corollary 2.2 says that the universal Riemannian covering $p: \quad \bar{X} \rightarrow X$ does not factor through a Riemannian covering $q: X^{\prime} \rightarrow X$ such that $X^{\prime}$ is the Riemannian product of a circle with another space. In particular, (i) is automatic if the Euclidean factor $X_{0}$ of $\bar{X}$ is reduced to a point.

Condition (ii) can be checked from the list [12, Theorem 6.1(4)]. There note that if $X_{i}$ is noncompact, say with compact dual $X_{i}^{*}$, then $W_{X_{i}}=W_{X_{i}^{*}}$.

\section{REFERENCES}

1. É. Cartan, Sur la détermination d'un système orthogonal complet dans un espace de Riemann symmétrique clos, Rend. Circ. Mat. Palermo 53 (1929), 1-36.

2. I. M. Gelfand, Spherical functions on symmetric spaces, Doklady USSR, 70 (1950), $5-8$. 
3. A. Kleppner, Representations induced from compact subgroups, Amer. J. Math., 88 (1966), 544-552.

4. K. de Leeuw, and H. Mirkil, Rotation-invariant algebras on the $n$-sphere, Duke Math. J., 30 (1963), 667-672.

5. G. W. Mackey, On induced representations of groups, Amer. J. Math., 73 (1951), 576-592.

6. - Symmetric and antisymmetric Kronecker squares and intertwining numbers of induced representations of finite groups, Amer. J. Math., 75 (1953), 387-405.

7. I. E. Segal, The two sided regular representation of a unimodular locally compact group, Annals of Math., 51 (1950), 293-298.

8. A. Selberg, Harmonic analysis and discontinuous groups in weakly symmetric spaces, J. Indian Math. Soc., 20 (1956), 47-87.

9. J. A. Tirao, Self adjoint function spaces on Riemannian symmetric manifolds, Proc. Amer. Math. Soc., 24 (1970), 223-228.

10. E. P. Wigner, On representations of certain finite groups, Amer. J. Math., 73 (1941), 57-63.

11.

, Condition that the irreducible representations of a group, considered as representations of a subgroup, do not contain any representation of the subgroup more than once, "Spectroscopic and Group Theoretical Methods in Physics," Wiley, New York, 1968.

12. J. A. Wolf, Self-adjoint function spaces on Riemannian symmetric manifolds, Trans. Amer. Math. Soc., 113 (1964), 299-315.

13. - Spaces of Constant Curvature, McGraw Hill, New York, 1967.

Received October 13, 1970. The first author was partially supported by NSF Grant GP-12997. The second author was partially supported by NSF Grant GP-8007.

UNIVERSity OF CALIFORNIA, BERKELEy 


\section{PACIFIC JOURNAL OF MATHEMATICS}

\section{EDITORS}

\author{
H. SAMELSON \\ Stanford University \\ Stanford, California 94305 \\ C. R. HовBY \\ University of Washington \\ Seattle, Washington 98105
}

J. DUGUndis

Department of Mathematics

University of Southern California

Los Angeles, California 90007

RICHARD ARENS

University of California

Los Angeles, California 90024

\section{ASSOCIATE EDITORS}
E. F. BeCKenbaCH
B. H. NeUmanN
F. WOLF
K. YoshidA

\section{SUPPORTING INSTITUTIONS}

\author{
UNIVERSITY OF BRITISH COLUMBIA \\ CALIFORNIA INSTITUTE OF TECHNOLOGY \\ UNIVERSITY OF CALIFORNIA \\ MONTANA STATE UNIVERSITY \\ UNIVERSITY OF NEVADA \\ NEW MEXICO STATE UNIVERSITY \\ OREGON STATE UNIVERSITY \\ UNIVERSITY OF OREGON \\ OSAKA UNIVERSITY \\ UNIVERSITY OF SOUTHERN CALIFORNIA
}

\author{
STANFORD UNIVERSITY \\ UNIVERSITY OF TOKYO \\ UNIVERSITY OF UTAH \\ WASHINGTON STATE UNIVERSITY \\ UNIVERSITY OF WASHINGTON

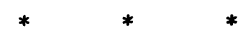 \\ AMERICAN MATHEMATICAL SOCIETY \\ CHEVRON RESEARCH CORPORATION \\ NAVAL WEAPONS CENTER
}

The Supporting Institutions listed above contribute to the cost of publication of this Journal, but they are not owners or publishers and have no responsibility for its content or policies.

Mathematical papers intended for publication in the Pacific Journal of Mathematics should be in typed form or offset-reproduced, (not dittoed), double spaced with large margins. Underline Greek letters in red, German in green, and script in blue. The first paragraph or two must be capable of being used separately as a synopsis of the entire paper. The editorial "we" must not be used in the synopsis, and items of the bibliography should not be cited there unless absolutely necessary, in which case they must be identified by author and Journal, rather than by item number. Manuscripts, in duplicate if possible, may be sent to any one of the four editors. Please classify according to the scheme of Math. Rev. Index to Vol. 39. All other communications to the editors should be addressed to the managing editor, Richard Arens, University of California, Los Angeles, California, 90024.

50 reprints are provided free for each article; additional copies may be obtained at cost in multiples of 50 .

The Pacific Journal of Mathematics is published monthly. Effective with Volume 16 the price per volume (3 numbers) is $\$ 8.00$; single issues, $\$ 3.00$. Special price for current issues to individual faculty members of supporting institutions and to individual members of the American Mathematical Society: $\$ 4.00$ per volume; single issues $\$ 1.50$. Back numbers are available.

Subscriptions, orders for back numbers, and changes of address should be sent to Pacific Journal of Mathematics, 103 Highland Boulevard, Berkeley, California, 94708.

PUBLISHED BY PACIFIC JOURNAL OF MATHEMATICS, A NON-PROFIT CORPORATION

Printed at Kokusai Bunken Insatsusha (International Academic Printing Co., Ltd.), 7-17, Fujimi 2-chome, Chiyoda-ku, Tokyo, Japan. 


\section{Pacific Journal of Mathematics}

Vol. 38, No. $2 \quad$ April, 1971

Richard Davis Anderson and Thomas Ashland Chapman, Extending

homeomorphisms to Hilbert cube manifolds .................. 281

Nguyen Huu Anh, Restriction of the principal series of $\operatorname{SL}(n, \mathbf{C})$ to some

reductive subgroups................................ 295

David W. Boyd, Indices for the Orlicz spaces . . . . . . . . . . . . 315

William Garfield Bridges, The polynomial of a non-regular digraph ...... 325

Billie Chandler Carlson, Robert K. Meany and Stuart Alan Nelson, Mixed

arithmetic and geometric means........................ 343

H. A. Çelik, Commutative associative rings and anti-flexible rings ...... 351

Hsin Chu, On the structure of almost periodic transformation groups ...... 359

David Allyn Drake, The translation groups of n-uniform translation

Hjelmslev planes ................................ 365

Michael Benton Freeman, The polynomial hull of a thin two-manifold . . . . 377

Anthony Alfred Gioia and Donald Goldsmith, Convolutions of arithmetic

functions over cohesive basic sequences .................... 391

Leslie C. Glaser, A proof of the most general polyhedral Schoenflies

conjecture possible ................................

Thomas Lee Hayden and Ted Joe Suffridge, Biholomorphic maps in Hilbert

space have a fixed point ................................ 419

Roger Alan Horn, Schlicht mappings and infinitely divisible kernels ...... 423

Norman Ray Howes, On completeness ...................... 431

Hideo Imai, Sario potentials on Riemannian spaces................ 441

A. A. Iskander, Subalgebra systems of powers of partial universal

algebras.

Barry E. Johnson, Norms of derivations of $\mathscr{L}(\mathrm{X})$.

David Clifford Kay and Eugene W. Womble, Axiomatic convexity theory and relationships between the Carathéodory, Helly, and Radon numbers

Constantine G. Lascarides, A study of certain sequence spaces of Maddox

and a generalization of a theorem of Iyer .............

C. N. Linden, On Blaschke products of restricted growth .

John S. Lowndes, Some triple integral equations ................. 515

Declan McCartan, Bicontinuous preordered topological spaces ......... 523

S. Moedomo and J. Jerry Uhl, Jr., Radon-Nikodým theorems for the Bochner and Pettis integrals ...

Calvin Cooper Moore and Joseph Albert Wolf, Totally real representations

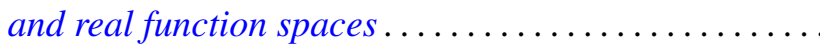

Reese Trego Prosser, A form of the moment problem for Lie groups. ... 